\title{
Do All Integrable Evolution Equations Have the Painlevé Property?
}

\author{
K.M. TAMIZHMANI ${ }^{\dagger}$, Basil GRAMMATICOS ${ }^{\ddagger}$ and Alfred RAMANI ${ }^{\S}$ \\ $\dagger$ Departement of Mathematics, Pondicherry University, Kalapet, 605014 Puducherry, India \\ E-mail:tamizh@yahoo.com \\ ‡IMNC, Université Paris VII-Paris XI, CNRS, UMR 8165, Bât. 104, 91406 Orsay, France \\ E-mail: grammaticos@univ-paris-diderot.fr \\ $\S$ Centre de Physique Théorique, Ecole Polytechnique, CNRS, 91128 Palaiseau, France \\ E-mail: ramani@cpht.polytechnique.fr
}

Received June 12, 2007; Published online June 19, 2007

Original article is available at http://www.emis.de/journals/SIGMA/2007/073/

\begin{abstract}
We examine whether the Painlevé property is necessary for the integrability of partial differential equations (PDEs). We show that in analogy to what happens in the case of ordinary differential equations (ODEs) there exists a class of PDEs, integrable through linearisation, which do not possess the Painlevé property. The same question is addressed in a discrete setting where we show that there exist linearisable lattice equations which do not possess the singularity confinement property (again in analogy to the one-dimensional case).
\end{abstract}

Key words: integrability; linearisability; Painlevé property; singularity confinement

2000 Mathematics Subject Classification: 34A99; 35A21; 39A12

The Painlevé property has been quintessential in the domain of integrable systems. While the modern era of integrability was spurred by the derivation (or rediscovery in some cases [1]) of evolution equations integrable by spectral methods (S-integrable systems in the Calogero terminology [2]), the Painlevé approach has almost from the outset become an unmatched integrability test leading to the discovery of a slew of new integrable systems. The conjecture [3], formulated by Ablowitz and Segur (in collaboration with one of us, A.R.) and which came to be known as the ARS conjecture, states that: "Every ordinary differential equation which arises as a reduction of a completely integrable partial differential equation is of Painlevé type (perhaps after a transformation of variables)". This conjecture was soon improved thanks to Weiss and collaborators who managed to treat partial differential equations directly, without the constraint of considering reductions [4].

We can illustrate the situation through the classical example of the modified-KdV equation:

$$
u_{t}=u_{x x x}-6 u^{2} u_{x}
$$

We expand the solution around a singularity manifold $\phi(x, t)$ (the calculations are very simple so there is no need to use the simplifying Kruskal ansatz [5]). We find the expansion

$$
u= \pm\left(\phi_{x} / \phi-\phi_{x x} \phi_{x}^{-1} / 2+\left(\left(\phi_{x x x}-\phi_{t}\right) \phi_{x}^{-2} / 6-\phi_{x x}^{2} \phi_{x}^{-3} / 4\right) \phi+c_{2} \phi^{2}+c_{3} \phi^{3}+\cdots\right)
$$

which is obviously singlevalued, and possesses the full complement of integration constants, satisfying thus the Painlevé property.

The discrete analogue of the Painlevé property is singularity confinement. As conjectured [6] by two of the authors (B.G and A.R.) in collaboration with V. Papageorgiou "any singularity spontaneously appearing in an integrable discrete system must disappear after a few iteration 
steps". (The fact that singularity confinement does not suffice as a discrete integrability criterion and must be complemented by the finiteness of the Nevanlinna order [7] of the solution has been discovered in [8] and was amply commented in several publications $[9,10])$. The discrete analogue of the $\mathrm{mKdV}$ equation

$$
x_{m+1, n+1}=x_{m, n} \frac{x_{m+1, n}-c x_{m, n+1}}{c x_{m+1, n}-x_{m, n+1}},
$$

where $c$ is a constant, has a singularity whenever, due to specific initial conditions, $x$ vanishes or becomes infinite. However this singularity disappears in the next iteration step and does not propagate at all: it is immediately confined.

An impressive number of examples have by now established the fact that both partial differential and difference equations integrable through spectral methods possess the Painlevé property, (or equivalently, have confined singularities). These results can be extended in a straightforward way to the case of ordinary equations (both differential and difference ones, this last family encompassing $q$-difference equations as well). This is a quite natural result since many ordinary equations can be obtained as reductions of partial ones. However it is in the domain of ordinary equations that exceptions to the reciprocal of the above statement have been obtained. Indeed, we found in [11] that there exist equations which are integrable through linearisation and which do not possess the Painlevé property. This is true both in the continuous and the discrete case. We shall illustrate this through two examples.

We start from the linear equation

$$
\frac{t x^{\prime \prime}+(a t-1 / 2) x^{\prime}+b t x}{x^{\prime \prime}+a x^{\prime}+b x}=K
$$

and take its derivative so as to eliminate $K$, obtaining a third order equation. Next we show that the same third order equation can be obtained if we start from the nonlinear equation

$$
x^{\prime \prime} x^{\prime}+2 a x^{\prime 2}+3 b x^{\prime} x+\left(2 a b-b^{\prime}\right) x^{2}=M
$$

and take its derivative so as to eliminate $M$. Here $a$ and $b$ are not free. We have $b=a^{2}-a^{\prime} / 2$ and $a$ satisfying the equation

$$
a^{\prime \prime \prime}=6 a^{\prime \prime} a+7 a^{\prime 2}-16 a^{\prime} a^{2}+4 a^{4}
$$

which is equation XII in the Chazy classification [12]. So, equation (2) is integrable by linearisation through equation (1). It is straightforward to show that (2) violates the Painlevé property. Solving it for $x^{\prime \prime}$, we find terms proportional to $x^{2} / x^{\prime}$ (and $1 / x^{\prime}$ ) which were shown to be incompatible with the Painlevé property [13].

A caveat is in order at this point. While there exist large classes of linearisable equations without the Painlevé property, there does also exist a family of linearisable equations which do satisfy the Painlevé criterion. The best known example of equations belonging to this class are the Riccati equation and its higher order analogues [14].

We turn now to an example of a discrete equation and examine the mapping

$$
x_{n+1}=a x_{n-1} \frac{x_{n}-a}{x_{n}-1} .
$$

As shown in [15], two singularities exist when either $x=1$ or $x=a$. The first singularity is confined leading to a finite singularity pattern $\{1, \infty, a\}$. The second singularity never confines unless $a=1$ (in which case the mapping is trivial) or $a$ is a cubic root of unity (with the resulting mapping being periodic with period six). 
On the other hand, (3) is linearisable. Indeed, introducing $y_{n}=x_{n} x_{n-1}-x_{n}-a x_{n-1}$ we reduce (3) to the linear mapping $y_{n+1}=a y_{n}$.

Of course the remark concerning the existence of linearisable systems with the Painlevé property has its analogue here for linearisable mappings with confined singularities: all mappings of the "projective" family fall into this class [16].

It is thus natural given the results presented above on PDE's integrable through spectral methods which possess the Painlevé property, and linearisable ODE's without it, to wonder whether linearisable PDE's without the Painlevé property may exist. Calogero has stressed the importance of the existence of PDE's integrable by methods different from the spectral ones. He has dubbed the members of this class C-integrable systems. So, quite understandably, we sought linearisable PDE's among the lengthy list of C-integrable systems established by Calogero [17]. We shall not go into a fully detailed analysis of the equations of the Calogero classification, but present just the outcome of our investigation. It turned out that the C-integrable equations presented by Calogero can be shown to belong to one of two classes.

The first class comprises equations which can be reduced to a linear one thanks to some transformation involving the integral of the variable of the nonlinear equation. A typical example is:

$$
u_{t}-u_{x x x}=3 u_{x x} u^{2}+9 u_{x}^{2} u+3 u_{x} u^{4}
$$

that is linearised to

$$
v_{t}-v_{x x x}=0
$$

through

$$
v(x, t)=u(x, t) \exp \int^{x} u\left(x^{\prime}, t\right)^{2} d x^{\prime} .
$$

Of course, the fact that the integral of $u^{2}$ appears in the transformation is not creating problems. As a matter of fact, it is possible to rewrite (5) as

$$
\frac{v_{x}}{v}=\frac{u_{x}}{u}+u^{2}=\frac{w_{x}}{2 w}+w
$$

which is just a Riccati equation for $w=u^{2}$. Quite expectedly, equation (4) (and others, similar to it) possess the Painlevé property.

The second class of the C-integrable systems of Calogero comprises equations which are obtained from some other integrable (sometimes linear) equations through hodograph transformations. The prototypical equation of this class is the Dym equation [2]

$$
u_{t}=u^{3} u_{x x x}
$$

which is related to the KdV equation. Equations of this class quite often possess the 'weak' Painlevé property. This is the case for the Dym equation. The expansion around a singularity manifold $\phi(x, t)$ is

$$
u_{0} \phi(x, t)^{2 / 3}+\sum_{p=1}^{\infty} u_{p} \phi(x, t)^{(p+2) / 3} .
$$

Moreover, there exist equations in the Calogero list of C-integrable PDE's belonging to the class of solvable through hodograph transformations which do not satisfy the Painlevé property at all. An example of such an equation is $u_{t}=f\left(u_{x}\right) / u_{x x}+g\left(u_{x}\right)+u h\left(u_{x}\right)$, where $f, g$ and $h$ are 
arbitrary functions. However due to the very special nature of the hodograph transformation we consider that such examples do not constitute a satisfactory answer to our quest.

So the question remains: do linearisable PDE's without the Painlevé property exist? The answer to this question is an unqualified "yes". Let us construct a specific example. We shall adopt the construction we follow for the derivation of the Burgers' equation. For the latter, we start from a linear equation $v_{t}+v_{x x}=0$ and obtain a nonlinear one through a Cole-Hopf relation $v_{x}+u v=0$. In order to derive the equation we are seeking, we start from a nonlinear, linearisable (Riccati) equation in one variable $v_{t}+v^{2}=0$ and couple it through a Cole-Hopf like relation to another variable in a new direction $u_{x}+u v=0$. Eliminating $v$ we obtain a nonlinear equation for $u$ :

$$
u u_{x t}-u_{x} u_{t}-u_{x}^{2}=0
$$

This is obviously a linearisable equation since its solution proceeds through the solution of one linearisable equation and a linear one, in cascade. The solution of this equation does not possess the Painlevé property. Instead of performing a standard Painlevé analysis let us profit from the fact that the solution of (6) can be explicitly constructed. Solving the equation for $v$ we find $v=(t-\phi(x))^{-1}$. Next we integrate for $u$ and obtain $\log u=-\int(t-\phi(x))^{-1} d x$. A singularity will appear in the expansion of $u$ whenever we have $x=\xi$ such that $\phi(\xi)=t$. We solve for $\xi$ and find $\xi=\psi(t)$ (where $\psi$ is the inverse function of $\phi$ ). Expanding $\phi(x)$ around $\xi$ we have $\phi(x)=\phi(\xi)+(x-\xi) \phi^{\prime}(\xi)+\cdots$ and the integration for $u$ can be performed order by order. We find $u \propto(x-\psi(t))^{\psi^{\prime}(t)}+\cdots$. Thus, since the exponent $\psi^{\prime}(t) \equiv 1 / \phi^{\prime}(\psi(t))$ is arbitrary, the solution does not possess the Painlevé property.

Equation (6) may be easily generalised. The principle remains the same. One starts from a linearisable equation in one independent and one dependent variable, say $v(t)$. If, for instance, we take for $v$ a higher-order projective equation, we are guaranteed that the solution for $v$ will satisfy the Painlevé property. Next we couple this equation to a linear PDE of the form $f(v) u_{x}+g(v) u_{t}+h(v) u=0$, where $f, g$, and $h$ can be taken as inhomogeneous linear functions of $v$. Eliminating $v$ one obtains an equation for $u$ which is linearisable and can be shown to violate the Painlevé property, the exponent of the leading singular term being again an arbitrary function of $t$.

We now turn to the case of a lattice linearisable equation. We start with a simple homographic mapping (the index $m$ is dummy at this level)

$$
v_{m, n+1}+1+\frac{1}{v_{m, n}}=0
$$

and couple it to a linear equation

$$
u_{m+1, n}-u_{m, n} v_{m, n}=0 .
$$

Eliminating $v$ we find for $u$ the equation

$$
u_{m+1, n+1} u_{m+1, n}+u_{m, n+1} u_{m+1, n}+u_{m, n} u_{m, n+1}=0 .
$$

While this equation is linearisable, it does not have confined singularities. Indeed, if at some lattice position we have $u_{m, n}=0$ (which is perfectly possible given the appropriate initial conditions) iterating (7) we find that $u_{k, n}=0$ for all $k \geq m$. On the other hand, since (7) is linearisable, we expect the growth of the sequence of its iterates to be linear. This turns out to be indeed the case. Taking initial conditions $u_{0,0}=$ const, $u_{0, n}=a(n)+b(n) p / q$, $u_{m, 0}=c(m)+f(m) r / s$ (with $a, b, c, f$ arbitrary functions of their argument) and computing the global homogeneous degree $d_{m, n}$ in $p, q, r, s$, we find that $d_{m, n}=m+2$ for $m>0$. 
Generalising (7) is quite straightforward. It suffices to start from a linearisable equation for $v$ of higher order (of which several examples do exist). Next we couple $v$ to a linear equation of the form $f\left(v_{m, n}\right) u_{m+1, n}+g\left(v_{m, n}\right) u_{m, n+1}+h\left(v_{m, n}\right) u_{m, n}=0$ where $f, g, h$ are first degree in $v$, and using the first equation we eliminate $v$. We surmise that the equation for $u$ will in general have unconfined singularities. However this has to be examined on a per case basis since there does not seem to exist a general argument for the singularity structure of the final equation.

The results we presented above can be further generalised. As a matter of fact, we do not have to take for the first equation one that satisfies the Painlevé property, or possesses confined singularities. It suffices that it be linearisable for our construction to go through (although here the Painlevé property, continuous or discrete, would be already violated at the first step). For the continuous case, we start from a linearisable equation in one dimension

$$
v_{t t}=\left(v_{t}-1\right) / v
$$

which is linearisable through the system $v_{t}=y v+1, y_{t}+y^{2}=0$. The general solution of (8) is $v=\left(c_{1}(x)+\log \left(t+c_{2}(x)\right)\left(t+c_{2}(x)\right)\right.$, which clearly violates the Painlevé property. (A singular solution for (8) also exists: $\left.v=t+c_{2}(x)\right)$. Next we couple the equation for $v$ to an equation for $u$ through $u_{x}-u v=0$ and obtain

$$
u_{x t t}=u_{x t}\left(2 \frac{u_{t}}{u}+\frac{u}{u_{x}}\right)+\frac{u_{t t} u_{x}}{u}-2 \frac{u_{x} u_{t}^{2}}{u^{2}}-u_{t}-\frac{u^{2}}{u_{x}}
$$

which is linearisable without having the Painlevé property. Turning to the discrete case we consider the one-dimensional mapping (again, the index $m$ is dummy at this level)

$$
y_{m, n+1}=y_{m, n}+y_{m, n}^{2} / y_{m, n-1}
$$

which is obtained from the linearisable system $z_{n+1}=z_{n}+1$ and $y_{n+1}=z_{n} y_{n}$. The solution of $(9)$ is $A(m) \Gamma(n+c(m)) \equiv B(m)(-1)^{n} / \Gamma(1-n-c(m))$ with $\pi A(m)=B(m) \sin (c(m) \pi)$. This solution is regular unless $c(m)$ is an integer. If $c(m)=N$, an integer, the first expression, with nonzero $A$, has poles on a half-infinite line $n \leq-N$, and is regular for $n>-N$. The second expression, for finite $B$, is regular for $n \leq-N$ and zero for $n>-N$. In both cases we have a typical nonconfined singularity. Coupling equation (9) with $x_{m+1, n}-y_{m, n} x_{m, n}=0$ leads to

$$
x_{m+1, n+1} x_{m+1, n-1} x_{m, n}^{2}=x_{m+1, n-1} x_{m+1, n} x_{m, n+1} x_{m, n}+x_{m+1, n}^{2} x_{m, n+1} x_{m, n-1} .
$$

Again, we have a linearisable equation with unconfined singularities. Many more examples can be explicitly constructed underlining the fact that there exist linearisable PDE's which do not satisfy the Painlevé property.

\section{References}

[1] Zabusky N.J., Kruskal M.D., Interaction of solitons in a collisionless plasma and the recurrence of ther initial state, Phys. Rev. Lett. 15 (1965), 240-243.

[2] Calogero F., Degasperis A., Spectral transforms and solitons, North-Holland, Amsterdam 1982.

[3] Ablowitz M.J., Ramani A., Segur H., Nonlinear evolution equations and ordinary differential equations of Painlevé type, Lett. Nuov. Cim. 23 (1978), 333-338.

[4] Weiss J., Tabor M., Carnevale G., The Painlevé property for partial differential equations, J. Math. Phys. 24 (1983), 522-526.

[5] Jimbo M., Kruskal M.D., Miwa T., Painlevé test for self-dual Yang-Mills equation, Phys. Lett. A 92 (1982), 59-60.

[6] Grammaticos B., Ramani A., Papageorgiou V., Do integrable mappings have the Painlevé property?, Phys. Rev. Lett. 67 (1991), 1825-1828. 
[7] Ablowitz M.J., Halburd R., Herbst B., On the extension of the Painlevé property to difference equations, Nonlinearity 13 (2000), 889-905.

[8] Hietarinta J., Viallet C.M., Singularity confinement and chaos in discrete systems, Phys. Rev. Lett. 81 (1991), 325-328.

[9] Grammaticos B., Tamizhmani T., Ramani A., Tamizhmani K.M., Growth and integrability in discrete systems, J. Phys. A: Math. Gen. 34 (2001), 3811-3821.

[10] Hietarinta J., Viallet C.M., Searching for integrable lattice maps using factorization, arXiv:0705.1903.

[11] Ramani A., Grammaticos B., Tremblay S., Integrable systems without the Painlevé property, J. Phys. A: Math. Gen. 33 (2000), 3045-3052.

[12] Chazy J., Sur les équations différentielles du troisième ordre et d'ordre supérieur dont l'intégrale générale à ses points critiques fixes, Acta Math. 34 (1911), 317-385.

[13] Ince E.L., Ordinary differential equations, Dover, London, 1956.

[14] Ramani A., Grammaticos B., Tamizhmani K.M., Lafortune S., Again, linearisable mappings, Physica A 252 (1998), 138-150.

[15] Tsuda T., Ramani A., Grammaticos B., Takenawa T., A class of integrable and nonintegrable mappings and their dynamics, Preprint, 2007.

[16] Ramani A., Grammaticos B., Karra G., Linearisable mappings, Physica A 181 (1992), 115-127.

[17] Calogero F., Why are certain nonlinear PDEs both widely applicable and integrable?, in What is Integrability?, Editor V.E. Zakharov, Springer, New York, 1990, 1-62. 